\title{
Disruption of CXCR3 function impedes the development of Sjögren's syndrome-like xerostomia in non-obese diabetic mice
}

\author{
Jing Zhou ${ }^{1,2} \cdot$ Qing $\mathrm{Yu}^{1,2}$
}

Received: 18 July 2017 / Revised: 7 November 2017 / Accepted: 5 December 2017 / Published online: 18 January 2018

(c) United States \& Canadian Academy of Pathology 2018

\begin{abstract}
The chemokine receptor CXCR3 plays an important role in $\mathrm{T}$ cell recruitment in various immune responses and autoimmune diseases. Expression of CXCR3 ligands, including CXCL9, CXCL10, and CXCL11, is elevated in the salivary glands of patients with Sjögren's syndrome (SS). To elucidate whether interaction between CXCR3 and its ligands is required for the development of SS, we administrated an anti-CXCR3 blocking antibody (CXCR3-173) to the non-obese diabetic (NOD) mice, a well-defined model of SS, during the stage prior to disease onset. Treatment with this anti-CXCR3 antibody significantly improved salivary secretion, indicating a remission of SS clinical manifestation. Anti-CXCR3 treatment did not affect the gross leukocyte infiltration of the submandibular glands (SMGs) as assessed by hematoxylin and eosin staining. However, flow cytometric analysis showed that anti-CXCR3 treatment markedly reduced the percentage of $\mathrm{CXCR} 3{ }^{+} \mathrm{CD} 8 \mathrm{~T}$ and $\mathrm{CXCR} 3{ }^{+} \mathrm{CD} 44^{+} \mathrm{CD} 8 \mathrm{~T}$ cells, without affecting that of $\mathrm{CXCR} 3{ }^{+} \mathrm{CD} 4 \mathrm{~T}$ and $\mathrm{CXCR} 3{ }^{+} \mathrm{CD} 44^{+} \mathrm{CD} 4 \mathrm{~T}$ cells in the SMGs and submandibular lymph nodes, suggesting a preferential effect of this anti-CXCR3 treatment on CXCR3-expressing effector CD8 T cells. Meanwhile, SMG expression of inflammatory factor TNF- $\alpha$ was markedly diminished by anti-CXCR3 treatment. In accordance, anti-CXCR3 significantly enhanced SMG expression of tight junction protein claudin-1 and water channel protein aquaporin 5, two molecules that are crucial for normal salivary secretion and can be down-regulated by TNF- $\alpha$. Taken together, these findings demonstrated that the interaction between the endogenous CXCR3 and its ligands plays a pro-inflammatory and pathogenic role in the development of SS-like xerostomia in the NOD mouse model.
\end{abstract}

\section{Introduction}

Sjögren's syndrome (SS) is a prevalent autoimmune disease that affects millions of people worldwide, predominantly women [1-3]. A characteristic pathology of this disease is leukocyte infiltration of salivary and lacrimal glands, leading to chronic inflammation, tissue destruction and secretory hypofunction [4-6]. Autoreactive effector T cells play an essential role in the pathogenesis of this disease [7-10] and the migration of these cells into exocrine glands

Qing Yu

qyu@forsyth.org

1 The Forsyth Institute, 245 First Street, Cambridge, MA 02142, USA

2 Department of Oral Medicine, Infection and Immunity, Harvard School of Dental Medicine, Boston, MA, USA requires the interaction between $\mathrm{T}$ cell-expressed chemokine receptors and with their specific ligands [11].

CXCR3 (C-X-C Motif Chemokine Receptor 3), a G protein coupled chemokine receptor, is absent in resting $\mathrm{T}$ cells and preferentially expressed by $\mathrm{T}$ helper 1 (Th1) $\mathrm{CD}^{+}$ $\mathrm{T}$ cell and $\mathrm{T}$ cytotoxic 1 (Tc1) $\mathrm{CD}^{+} \mathrm{T}$ cells [12]. CXCR3 can bind to and be activated by its ligands CXCL9 (chemokine induced by IFN- $\gamma$ ), CXCL10 (interferon-inducible protein 10) and CXCL11 (interferon-inducible T-cell $\alpha$ chemoattractant) to orchestrate effector $\mathrm{T}$ cell generation in lymphoid tissues and their subsequent trafficking to peripheral inflammatory sites [13]. Expression of CXCL9, CXCL10, and CXCL11 can be up-regulated by pro-inflammatory cytokines, including IFN- $\gamma$ and TNF- $\alpha$, in a variety of cell types [13-15]. Consistent with this, our recent study demonstrated the down-regulation of CXCL9 in the salivary glands of an SS-like mouse model as a result of TNF- $\alpha$ blockade [16]. CXCR3 ligands play important roles in the pathogenesis of various inflammatory and autoimmune diseases [13,17-21]. Deficiency of CXCR3 leads to impaired infiltration of effector $\mathrm{T}$ cells into disease target 
organs and amelioration of autoimmune insulitis, diabetes and systemic lupus erythematosus (SLE) [22-24]. Inhibition of CXCR3 function with blocking antibodies or small molecule antagonists has shown a therapeutic effect in experimental models of $\mathrm{T}$ cell-mediated inflammatory diseases, including rheumatoid arthritis, alopecia areata, adjuvant arthritis and severe sepsis, with the effect mostly associated with inhibition of Th1 and Tc1 cell recruitment to the target organs [25-28]. In addition, a therapeutic regimen that blocks CXCL10-CXCR3 axis using a neutralizing anti-CXCL10 antibody has recently been tested in a randomized and double-blind clinical trial for rheumatoid arthritis and led to promising outcomes [29]. Collectively, CXCR3 and its ligands contribute to the pathogenesis of a variety of $\mathrm{T}$ celldependent inflammatory and autoimmune diseases.

The recruitment of CXCR3-expressing T cells and elevated CXCR3 ligands CXCL9, CXCL910, and CXCL911 produced by ductal epithelial cells have been detected in the salivary glands of SS patients and experimental mouse models of this disease [30-32], which suggests that CXCR3 expression on $\mathrm{T}$ cells may play a critical role in the migration of these cells into disease target organs and the initiation of local autoimmune responses. Consistent with this potential function of CXCR3, antagonism of CXCL10 activity in MRL/lpr mouse model of SS significantly impedes the progression of SS-like sialadenitis and ameliorates tissue destruction by reducing leukocyte infiltration of the salivary glands [33]. However, the function of overall CXCR3dependent pathways constituted by chemokine receptor CXCR3 and its three ligands in the pathogenesis of SS, particularly in the early stage of the development prior to disease onset, has not been characterized.

In the present study, we investigated the particular role of endogenous CXCR3-mediated pathways in the development of SS-like sialadenitis, using a neutralizing antibody targeting the receptor $\mathrm{CXCR} 3$, rather than the individual ligands, in nonobese diabetic (NOD) mice, a well-defined mouse model of $\mathrm{SS}$. We demonstrated that CXCR3 plays an indispensable role in the development of SS-like xerostomia.

\section{Materials and methods}

\section{Mice}

Female non-obese diabetic (NOD) mice were purchased from the Jackson Laboratory and were maintained in the specific pathogen-free animal facility at the Forsyth Institute. All the experimental protocols were approved by the Institutional Animal Care and Use Committee of the Forsyth Institute. All the procedures were performed in compliance with the National Institutes of Health guidelines for the care and use of laboratory animals.

\section{Antibodies}

Purified monoclonal anti-mouse CXCR3 (CXCR3-173) and its isotype control hamster IgG used for injection were obtained from BioXCell. For flow cytometry, anti-CD4, anti-CD8, anti-CD44, and anti-CXCR3 antibodies were purchased from BioLegend. For immunohistological chemistry, anti-TNF- $\alpha$ and anti-claudin-1 antibodies were purchased from Abcam. For immunofluorescence staining, anti-aquaporin-5 (AQP5) and Alexa Fluor647-conjugated rabbit IgG were purchased from Abcam.

\section{In vivo administration of anti-CXCR3 antibody}

Four-week-old female NOD mice received intraperitoneal (i. p.) administration of $200 \mu \mathrm{g}$ anti-mouse CXCR3 antibody or control hamster IgG 3 times weekly for 6 weeks. All the analyses were performed 2 days after the last injection.

\section{Histological, immunohistochemical and immunofluorescence staining}

Harvested SMG tissues were fixed in $4 \%$ paraformaldehyde, embedded in paraffin and sectioned to $5 \mu \mathrm{m}$ thickness. The sections were de-paraffinized and subsequently stained with hematoxylin and eosin $(\mathrm{H} \& \mathrm{E})$ to determine the degree of inflammation. For immunohistochemical staining, the deparaffinized sections were stained with antibodies against mouse TNF- $\alpha$ and claudin- 1 at $4{ }^{\circ} \mathrm{C}$ overnight using VECTASTAIN Elite ABC Kit (Vector Laboratories) following the manufacturer's manual. For immunofluorescence staining, the de-paraffinized sections were incubated with anti-AQP5 antibody after antigen retrieval. They were then incubated with Alexa Fluor 647-conjugated mouse IgG and imaged using a Leica laser scanning confocal microscope (Zeiss). ImageJ $1.50 \mathrm{i}$ software was used to quantify the positively stained areas of immunohistochemical staining and the fluorescence intensity of immunofluorescence staining in the sections.

\section{Flow cytometry}

Freshly isolated single cells from SMG or submandibular lymph nodes (smLNs) were first incubated with anti-CD16/ 32 antibody to block non-specific binding of immunoglobulin to Fc $\gamma$ III/II receptors. The cells were then stained with combinations of fluorescence-conjugated antibodies to CD4, CD8, CD44 and CXCR3 at $4{ }^{\circ} \mathrm{C}$ for $30 \mathrm{~min}$. After washing with cold PBS containing $1 \%$ FBS, all the stained samples were analyzed with a FACS Arial II flow cytometer (BD), and the raw data were calculated with the FlowJo V10 software. 


\section{Detection of serum antinuclear antibodies (ANA)}

ANA levels in mouse sera (1:40 dilution in PBS) were determined using HEp-2 human epithelial cell substrate slides (INOVA Diagnostics), according to the manufacturer's instructions. After staining, the images were acquired on an inverted wide-field fluorescence microscope (Zeiss) at $\times 400$ magnification. Images presented were processed using Zeiss software (ZEN blue edition).

\section{ELISA}

The custom synthesized M3R peptide fragment (Biomatik Corporation) containing 15 amino acids of the second extracellular loop of M3R was dissolved in PBS initially and further diluted to a final concentration of $2 \mu \mathrm{g} / \mathrm{ml}$ in the $1 \times$ ELISA coating buffer (BioLegend) immediately before use. The Nunc ${ }^{\mathrm{TM}}$ MaxiSorp ${ }^{\mathrm{TM}}$ flat-bottom 96 well plates (BioLegend) were coated with the M3R peptide solution at $4{ }^{\circ} \mathrm{C}$ overnight. Non-specific binding sites on the plates were blocked with ELISA Assay Diluent buffer (BioLegend). Sera (1:6 diluted) were then added to the plate and incubated over night at $4{ }^{\circ} \mathrm{C}$. After washing with $0.05 \%$ Tween 20 in PBS, the plates were incubated with 1:300 diluted biotinylated goat anti-mouse IgG antibody (Vector Laboratories) for $1 \mathrm{~h}$. Subsequently, the plates were incubated with avidinHRP solution for $30 \mathrm{~min}$ following removal of excess detection antibody. Finally, the bound antibodies on plates were detected by the TMB substrate and the reaction was stopped with $100 \mu \mathrm{l} 2 \mathrm{~N} \mathrm{H} 2 \mathrm{SO} 4$ each well. The absorbance at $450 \mathrm{~nm}$ was read with a microplate reader (BioTek).

\section{Measurement of stimulated salivary flow rate}

NOD mice received an i.p.-injection of $100 \mu l$ PBS-based secretagogue solution containing pilocarpine $(0.05 \mathrm{mg} / \mathrm{ml})$ and isoproterenol $(0.02 \mathrm{mg} / \mathrm{ml})$. One min after secretagogue administration, saliva was collected continuously for $5 \mathrm{~min}$ from the oral cavity of mice with a micropipette. The volume of saliva from each mouse was measured and normalized to the body weight. The measurement was consistently performed in the mid-afternoons to exclude the effect of circadian rhythm on the results.

\section{Real-time RT-PCR}

Total RNA was extracted using RNeasy Micro kit (Qiagen) and the complementary DNA (cDNA) was synthesized with MLV reverse transcriptase (Promega) according to the manufacturer's instructions. Subsequently, SYBR Green-based real-time PCR amplification (Qiagen) was performed for 40 cycles with annealing and extension temperature at $60^{\circ} \mathrm{C}$, on a LightCycler 480 Real-Time PCR System (Roche). Primer sequences are as follows: mouse CXCL9, forward, 5'CCCTCAAAGACCTCAAACAGT-3'; reverse, 5'-AGTCC GGATCTAGGCAGGTT-3'; CXCL10 forward, 5'-CCAGTGAGAATGAGGGCCAT-3', reverse, 5'-CCGGATTCAGACATCTCTGC-3'; CXCL11 forward, 5'-GCAGAGATC GAGAAAGCTTCT-3', reverse, 5'-GTCCAGGCACCTTT GTCGTT-3';. All transcript levels were normalized to $\beta$-actin.

\section{Statistical analysis}

The Student's $t$-test (two-tailed, two sample equal variance) was used to determine all statistical significance. $P$ values smaller than 0.05 were considered as statistically significant.

\section{Results}

\section{Expression of CXCR3 ligands in the submandibular glands (SMGs) of NOD mice}

Elevated expression of CXCR3 ligands, CXCL9, CXCL10 and CXCL11, is often detected in the target tissues of autoimmune diseases [17], including the SMGs of SS patients $[32,34]$. Here, we first determined whether the increased expression of these CXCR3-targeting chemokines in the SMGs accompanies the development of SS disease in female NOD mice. These mice used in our study have the disease initiation around 10 weeks of age as demonstrated by the presence of SMG-infiltrating leukocyte foci and serum ANA, as well as reduced salivary flow rate in majority of these mice compared to 4 week-old female NOD mice as well as 10 week-old control Balb/c mice (data not shown). We examined the mRNA levels of CXCR3 ligands in the SMGs of female NOD mice at 4, 7 and 10 weeks of age by real-time PCR analysis. The results showed that the mRNA amounts of all three CXCR3 ligands were significantly increased between 4 and 10 weeks of age (Fig. 1a). In contrast, CXCL10 and CXCL11 did not increase with between 4 and 10 weeks of age in the control Balb/c mice (Fig. 1b). Although CXCL9 did exhibit an age-dependent increase in the Balb/ c mice, the degree of increase (3.6 fold) was significantly smaller than that in the NOD mice (9.1 fold, Fig. 1b). Therefore, the increase in CXCR3 ligand levels in the NOD mice is mostly due to the SS disease development but not the aging process per se. Hence, CXCR3 ligand expression in the SMGs is increased accompanying the development of SS in the NOD mice, suggesting the 
A

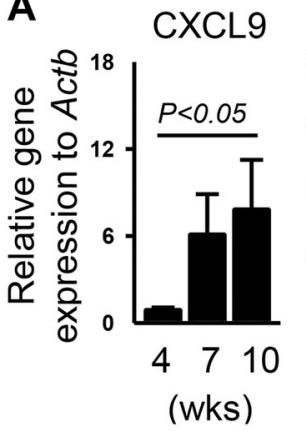

CXCL11

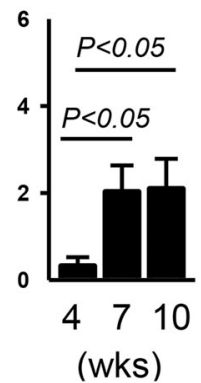

B

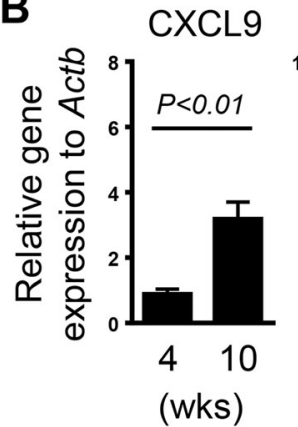

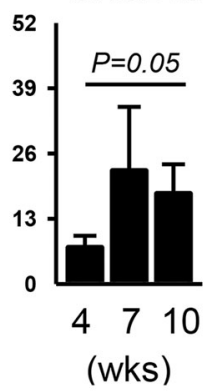

CXCL10

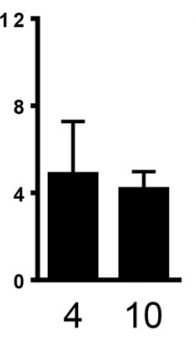

(wks)
CXCL11

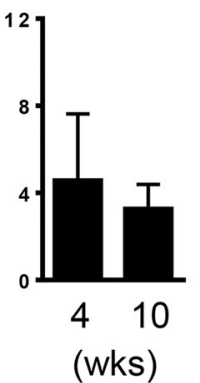

Fig. 1 Expression level of CXCR3 ligands in the SMGs of NOD and Balb/c mice. Real-time PCR analysis of CXCL9, CXCL10, and CXCL11 levels in the SMGs of NOD mice aged 4, 7, and 10 weeks (a) and those in the SMGs of Balb/c mice aged 4 and 10 weeks (b). The results are presented relative to that of $\beta$-actin. Data are the average of analyses of 4-7 mice for each group. Error bars represent the SEM

involvement of these CXCR3 ligands in the pathogenesis of this disease.

\section{In vivo administration of a blocking antibody against CXCR3 impedes the development of hyposalivation without affecting gross leukocyte infiltration of SMGs in NOD mice}

To determine whether CXCR3 ligand-CXCR3 interaction plays an important role in the development of SS, we i.p.injected $200 \mu \mathrm{g}$ of an anti-CXCR3 antibody, which blocks the interaction between CXCR3 and its ligands [35], or its isotype control into 4-week-old female NOD mice 3 times weekly for 6 weeks. Since impaired salivary secretion is one of the signature pathologies of SS, we measured this parameter and demonstrated that anti-CXCR3-treated mice had a higher stimulated salivary flow rate, normalized to the body weight, compared to that of IgG-treated controls (Fig. 2a). Hence, blockade of endogenous CXCR3 impedes the development of salivary gland secretory dysfunction in NOD mice.

We further assessed the effect of endogenous CXCR3 on leukocyte infiltration of SMGs, another pathological hallmark of SS. H\&E staining showed that the gross amount of leukocytes in the SMGs was not reduced by anti-CXCR3 treatment (Fig. 2b).

A

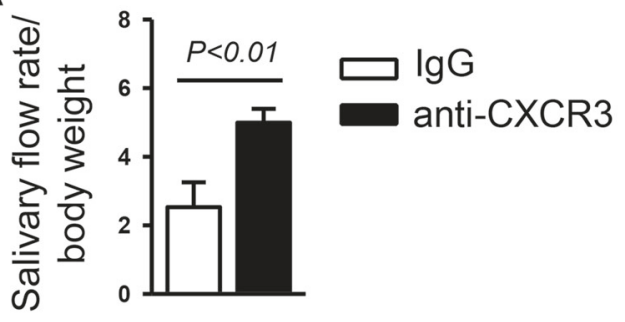

B

$\lg G$

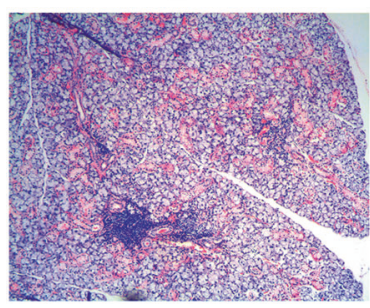

anti-CXCR3

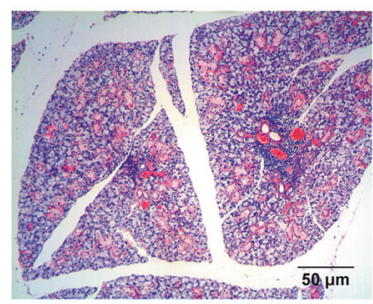

Fig. 2 Anti-CXCR3 treatment impedes the development of hyposalivation without affecting gross leukocyte infiltration of the SMGs in NOD mice. Anti-CXCR3 antibody or IgG was i.p.-administered to 4week-old female NOD mice 3 times weekly for 6 weeks. a The volume of saliva that was collected during the $5 \mathrm{~min}$ period, normalized to body weight. b Images of H\&E staining of SMG sections. Original magnification: $\times 200$. Data are representative or the average of 6-12 mice for each group

\section{Treatment with anti-CXCR3 reduces the frequency of CXCR3-expressing effector CD8 $T$ cells in the SMGs and submandibular lymph nodes (smLNs)}

Since CXCR3 plays an important role in the trafficking of effector T cells into inflamed peripheral tissues, we further investigated whether CXCR3 blockade could affect the CD4 and CD8 T cell subsets. Consistent with the H\&E results, flow cytometric analysis revealed that the percentage of CD4 and CD8 T cells in the SMGs was comparable between anti-CXCR3-treated and IgG-treated mice. However, CXCR3 blockade significantly reduced the frequency of $\mathrm{CXCR}^{+} \mathrm{CD} 8 \mathrm{~T}$ and $\mathrm{CXCR} 3{ }^{+} \mathrm{CD} 44^{+} \mathrm{CD} 8 \mathrm{~T}$ cells, whereas it did not alter that of $\mathrm{CXCR} 3{ }^{+} \mathrm{CD} 4 \mathrm{~T}$ or CXCR3 ${ }^{+} \mathrm{CD} 44^{+} \mathrm{CD} 4 \mathrm{~T}$ cells in the SMGs (Fig. 3a). Consistent with the findings in the SMGs, anti-CXCR3 treatment did not affect the percentage of total CD4 or CD8 $\mathrm{T}$ cells, but markedly reduced that of $\mathrm{CXCR}^{+} \mathrm{CD} 8 \mathrm{~T}$ and $\mathrm{CXCR} 3$ ${ }^{+} \mathrm{CD} 44^{+} \mathrm{CD} 8 \mathrm{~T}$ cells without affecting that of CXCR3 ${ }^{+} \mathrm{CD} 4 \mathrm{~T}$ and $\mathrm{CXCR} 3{ }^{+} \mathrm{CD} 44{ }^{+} \mathrm{CD} 4 \mathrm{~T}$ cells in the smLNs (Fig. 3b). The findings indicate that the accumulation of Tc1 cells, rather than Th1 cells is selectively inhibited in SS disease target organs as a result of CXCR3 blockade. In addition, we also investigated the effect of anti-CXCR3 on $\mathrm{B}$ cells, which together with $\mathrm{T}$ cells constitute the majority of infiltrating leukocytes in the SMGs $[1,36]$. The results demonstrated that the percentage of $\mathrm{B}$ cells, defined by 


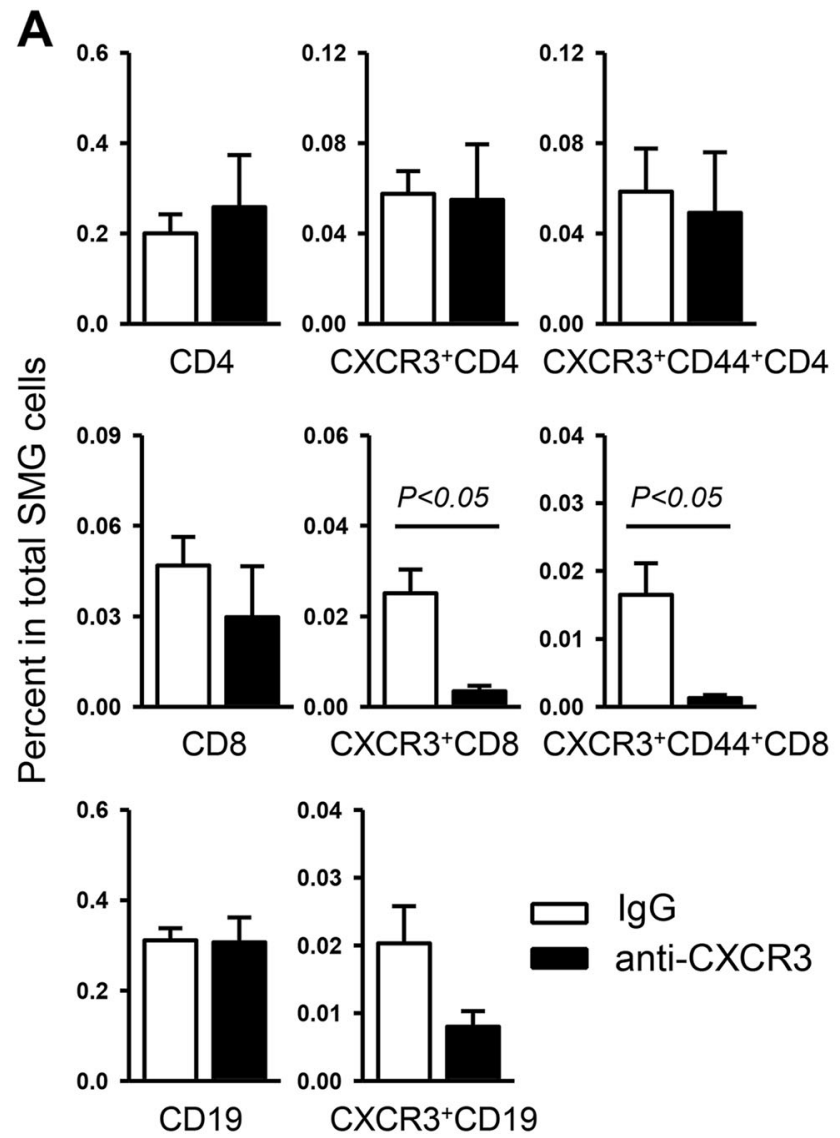

Fig. 3 Anti-CXCR3 treatment reduces the frequency of CXCR3expressing effector CD8 $\mathrm{T}$ cells in the SMGs and smLNs. AntiCXCR3 antibody or IgG was i.p.-administered to 4-week-old female

CD19 expression, in the SMGs was not changed by antiCXCR3 treatment in a statistically significant fashion (Fig. 3a). Hence, our findings suggest the preferential effect of this anti-CXCR3 antibody treatment on CD8 effector T cell accumulation/expansion in the SMGs and smLNs, which may contribute to the secretory dysfunction and pathogenesis of SS in NOD mice.

\section{Treatment with anti-CXCR3 reduces TNF-a expression and elevates claudin-1 and AQP5 expression in the SMGs}

Excessive pro-inflammatory factor TNF- $\alpha$ has been detected in both salivary glands and blood of SS patients [37,38] and plays a pathogenic role in SS development in the NOD model of SS disease [16]. To assess whether CXCR3 blockade can diminish TNF- $\alpha$ production, we performed immunohistochemical staining of SMG sections, which showed that anti-CXCR3 treatment significantly reduced the TNF- $\alpha$ protein level (Fig. 4a). Hence, the results imply that endogenous CXCR3 exerts tissue pro-inflammatory
B
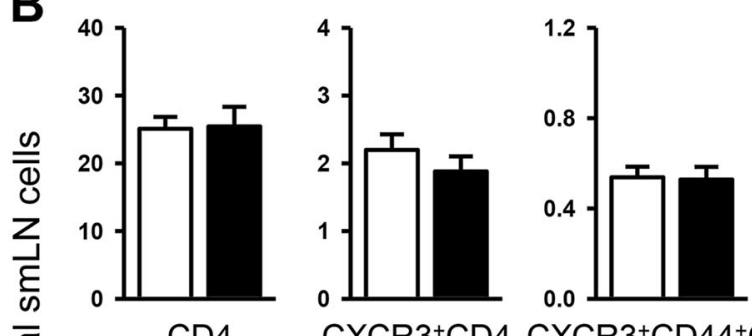

$\mathrm{CXCR}^{+} \mathrm{CD} 4 \mathrm{CXCR}^{+}{ }^{+} \mathrm{CD} 44^{+} \mathrm{CD} 4$
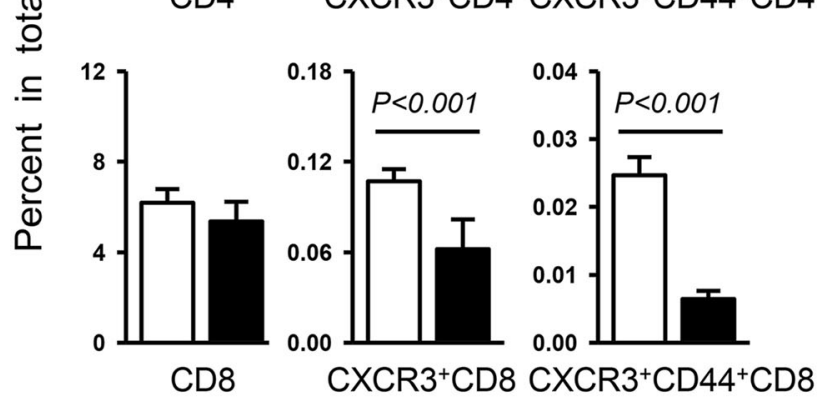

NOD mice 3 times weekly for 6 weeks. Flow cytometry of lymphocyte populations in the SMGs (a) and smLNs (b). Data are the average of analyses of 6-12 mice for each group

function through exacerbating TNF- $\alpha$ production during the development of SS disease.

Impaired tight junction integrity has been documented in the salivary glands of SS patients [39] and contributes to the secretory dysfunction of rat salivary gland epithelial cells [40]. To determine whether CXCR3 blockade affects the tight junction integrity in the SMGs, we examined the protein levels of claudin-1, claudin-2, and occludin in the SMGs by immunohistochemical staining and found that claudin-1 expression was significantly increased, whereas claudin-2 or occludin levels were not altered by antiCXCR3 treatment (Fig. 4b, and data not shown). The results indicate that the improved salivary secretion as a result of CXCR3 blockade is associated with elevated claudin-1, which is important for TJ structure and function [41]. To define additional potential molecular mechanisms of enhanced salivary secretion caused by anti-CXCR3 treatment, we measured the level of AQP5, a water channel protein that is crucial for normal salivary fluid secretion [42] $[43,44]$. By immunofluorescence staining of SMG sections, we found a markedly higher level of AQP5 expression in 
A TNF- $\alpha \mathrm{IHC}$
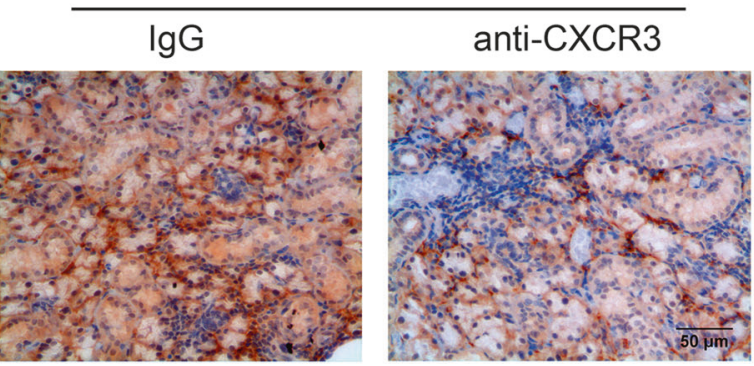

B
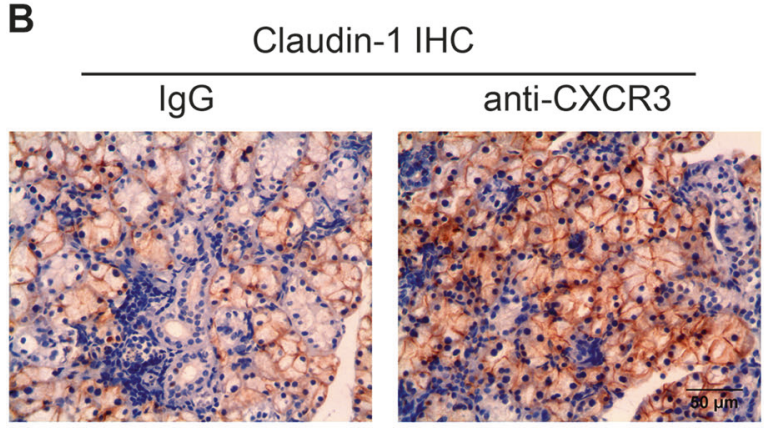

C
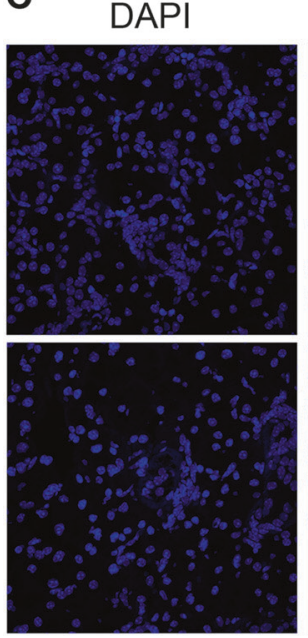
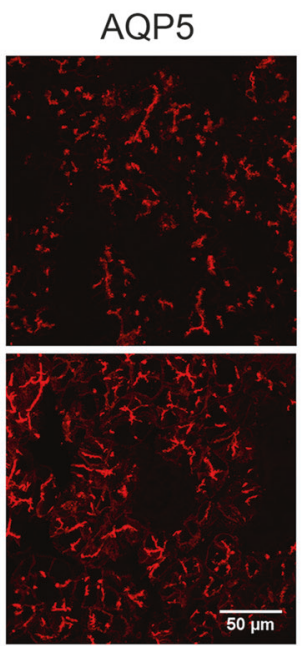

$\mathrm{DAPI}+\mathrm{AQP} 5$

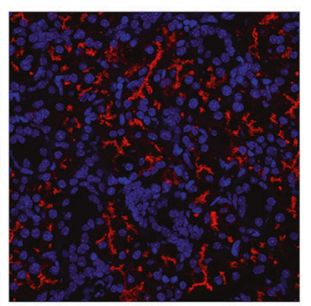

$\lg G$

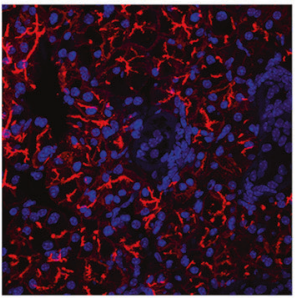

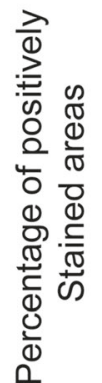
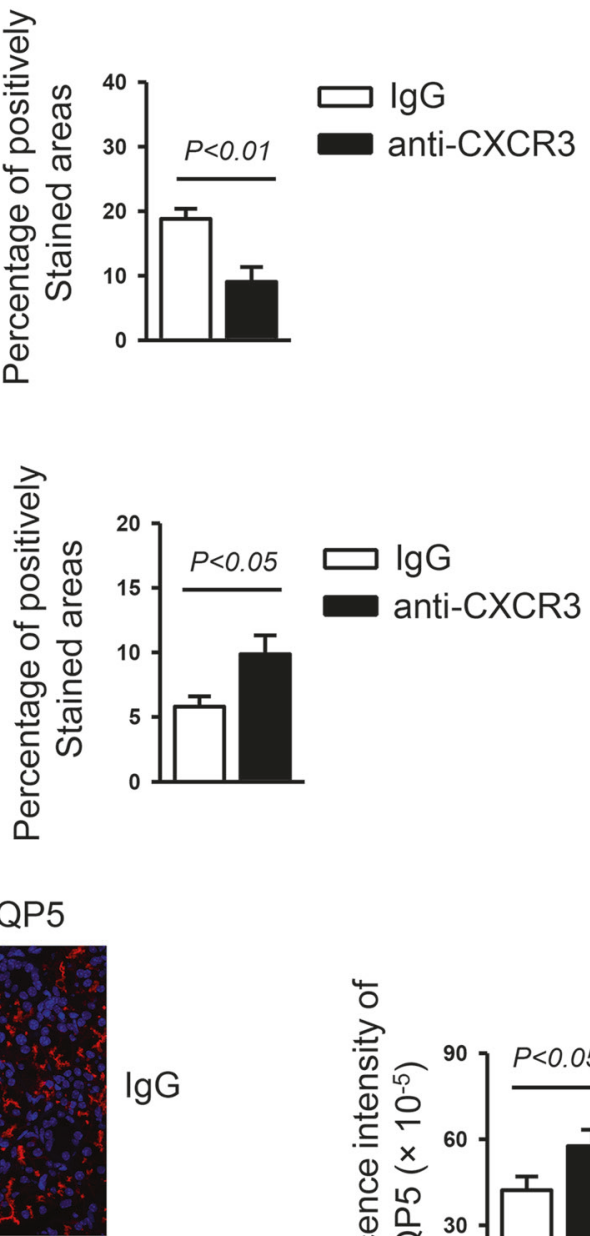

anti-CXCR3

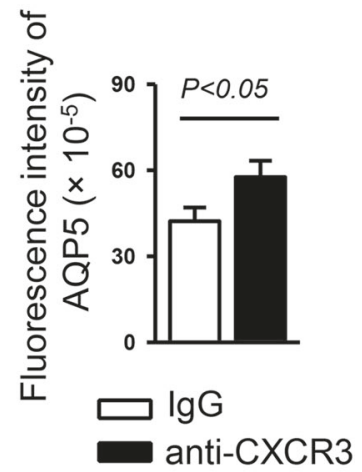

Fig. 4 Anti-CXCR3 treatment reduces TNF- $\alpha$ expression and elevates claudin-1 and AQP5 expression in the SMGs. Anti-CXCR3 antibody or IgG was i.p.-administered to 4-week-old female NOD mice 3 times weekly for 6 weeks. a Immunohistochemical staining of TNF- $\alpha$ protein in SMG sections. Bar graph shows the percentage of positively stained areas in the sections. b Immunohistochemical staining of claudin-1 protein in SMG sections. Bar graph shows the percentage of positively stained areas in the sections. c Immunofluorescence staining of AQP5 protein in SMG sections. Bar graph shows the fluorescence intensity of AQP5 staining. Data are representative or the average of analyses of 12-15 mice for each group. All images were captured with $\times 400$ original magnification

the mice with CXCR3 blockade than IgG treatment controls (Fig. 4c). Thus, the increased APQ5 expression may contribute to the improvement of salivary secretion induced by CXCR3 blockade.

\section{Anti-CXCR3 treatment leads to enhanced autoantibody production}

To explore whether CXCR3 blockade alters the production of autoantibodies, a characteristic pathological change of SS disease, we examined serum antinuclear antibody (ANA) levels by indirect immunofluorescence staining using human epithelial (HEp-2) cells as substrates. The results showed that anti-CXCR3 treatment led to a substantial increase in the level of ANA as indicated by higher fluorescence intensity of the staining compared to IgG-treated control group (Fig. 5a). We also measured the level of autoantibodies against M3 muscarinic acetylcholine receptor (M3R), which are important factors contributing to the secretory dysfunction of salivary gland in response to 
A

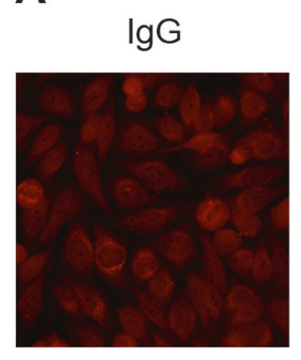

anti-CXCR3

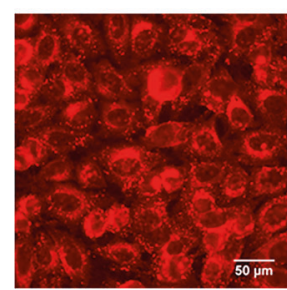

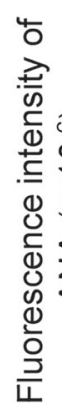

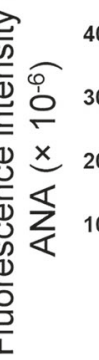

B

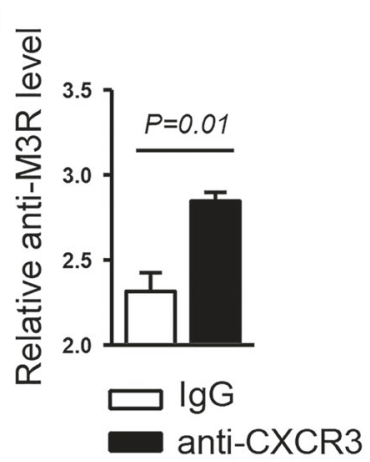

Fig. 5 Anti-CXCR3 treatment leads to enhanced autoantibody production. Anti-CXCR3 antibody or IgG was i.p.-administered to 4week-old female NOD mice 3 times weekly for 6 weeks. a Detection of serum ANA. Original magnification: $\times 400$. Bar graph shows the fluorescence intensity of ANA staining. b Analyses of serum anti-M3R autoantibody level by ELISA. Data are representative or the average of analyses of 12-15 mice for each group. neurotransmitters [2, 45]. Enzyme-linked immunosorbent assay (ELISA) demonstrated a higher level of anti-M3R antibodies in the sera from anti-CXCR3-treated mice than those from IgG-treated mice (Fig. 5b). Taken together, these findings showed that CXCR3 blockade leads to exacerbated autoantibody production during SS development, indicating its complex and multifaceted functions in this disease.

\section{Discussion}

This study demonstrated that endogenous CXCR3-mediated pathways play important pro-inflammatory and pathogenic roles in the development of SS-like siadadentis using NOD mice and provided several potential cellular and molecular mechanisms of CXCR3 function, including promotion of effector CD8 T cell accumulation, enhancement of TNF- $\alpha$ production, and down-regulation of tight junction protein claudin-1 and water channel protein AQP5 in the SMGs, a target tissue of SS.

The antibody clone targeting CXCR3 (CXCR3-173) used in our work has been reported to suppress $\mathrm{T}$ cell migration by inhibiting CXCR3-dependent pathways in multiple in vivo studies $[26,46]$. An in vitro study has demonstrated that CXCR3-173 inhibits the binding of CXCL10 and CXCL11 to CXCR3 [35]. It has also shown that the antibody has little inhibitory effect on the binding between CXCL9 and CXCR3, a claim that requires more rigorously designed assays to further confirm. Nevertheless, using this antibody, our study unequivocally demonstrated that the interaction between CXCR3 and its ligands plays a crucial role in the development of SS in the NOD model of this disease.

$\mathrm{T}$ cell trafficking into exocrine glands is a critical event for the pathogenesis of SS disease [1]. Studies of SS patients have reported the presence of elevated expression of T cell chemoattractants CXCL9, CXCL10, and CXCL11 in the salivary glands [31, 32]. In accordance with these findings, we showed that the expression of these three CXCR3-binding ligands in the SMGs of NOD mice increases with age accompanying SS development. By inhibiting CXCR3 function with a neutralizing anti-CXCR3 antibody, we demonstrated that CXCR3 is essential for the development of salivary secretory dysfunction in NOD mice. Moreover, anti-CXCR3 treatment preferentially reduces the number of CXCR3-expressing effector CD8 $\mathrm{T}$ cells in the SMGs and smLNs. Our results are consistent with a recent report showing that administration of the same anti-CXCR3 antibody clone to a mouse model of areata (AA), another autoimmune disease, prevents the recruitment of a subset of effector CD8 $\mathrm{T}$ cells to the target tissue sites and lymph nodes, and restrains the development of this disease [26]. Moreover, CXCR3-deficiency impairs the migration of effector CD8 $\mathrm{T}$ cells into the lungs in a lung transplantation rejection model [47]. Thus, CXCR3mediated pathways have a crucial function in effector CD8 $\mathrm{T}$ cell trafficking into the inflamed tissue sites under various disease conditions. In addition, our study did not find a significant effect of CXCR3 blockade on the total SMG-infiltrating mononuclear cells, T cells and Th1 cells, which is different from a previous report showing that CXCL10 antagonism in MRL-lpr model of SS markedly reduces the amount of total mononuclear cells, $\mathrm{T}$ cells and $\mathrm{CXCR}^{+} \mathrm{T}$ cells in the SMGs [33]. The difference may be in part attributed to the different targets of blockade. Compared to the antagonism of CXCL10 activity alone, our study used a neutralizing antibody targeting CXCR3 receptor rather than a single ligand, in order to reveal the function of CXCR3-mediated pathways in the SS development. Additionally, it may also be attributed to the differences in the disease pathogenesis and pathologies resulting from the different genetic and environmental factors in the two mouse models used. 
It is also worth noting that our study excluded the interference from the clinical type-1 diabetes, which generally develops after 12-16 weeks of age in female NOD mice, later than the initial onset of SS when the mice were analyzed in this study. Hence, the effect of anti-CXCR3 treatment on SS is not an indirect consequence of altered type-1 diabetes development.

Elevated level of pro-inflammatory factor TNF- $\alpha$ has been detected in the salivary glands of SS patients [38]. Our recent report provided evidence that $\mathrm{TNF}-\alpha$ is essential for the pathogenesis of SS through promoting CXCR3 ligand expression and facilitating $\mathrm{T}$ lymphocyte infiltration of the SMGs of NOD mice [16]. In the present work, we demonstrated that CXCR3 blockade in turn down-regulates TNF- $\alpha$ expression in the SMGs, indicative of a positive feed-forward loop formed between CXCR3 and TNF- $\alpha$ which amplifies salivary gland inflammation. Moreover, both TNF- $\alpha$ neutralization and CXCR3 blockade lead to increased claudin-1 and AQP5 levels in the SMGs [16], further suggesting a close interplay between TNF- $\alpha$ and CXCR3-mediated pathways. Claudin-1 is an essential component of intercellular tight junction, crucial for a well-regulated diffusion barrier between apical and basal regions of exocrine gland cells and the maintenance of water and solute exchange [41]. AQP5 is essential for normal salivary secretion and studies have shown that AQP5 deficiency significantly impairs secretory function due to defective water export in salivary gland epithelial cells and that local gene therapy with AQP1 overexpression can compensate for AQP5 deficiency [42-44]. Hence, these findings suggest that endogenous CXCR3-mediated pathways promote salivary secretory dysfunction, at least in part, through augmenting TNF- $\alpha$ production to down-regulate the expression of claudin-1 and AQP5.

One intriguing finding in this study is that administration of anti-CXCR3 antibody exacerbates the production of ANA and anti-M3R autoantibodies. We and other groups have reported the negative correlation between the autoantibody production and TNF- $\alpha$ pathway [16, 48,49]. We thereby speculate that the appearance of excessive autoantibodies caused by CXCR3 blockade is the consequence of diminished TNF- $\alpha$ production. Interestingly, anti-TNF- $\alpha-$ induced auto-antibodies in patients with SLE usually fall back to baseline levels shortly after the termination of antiTNF- $\alpha$ treatment, and thus only leads to transient and reversible clinical manifestation of this disease [49-51]. The exact function and potential mechanism of the excessive autoantibody production resulting from the CXCR3 blockade during SS development await to be further investigated.

Finally, the pathogenic functions of CXCR3 and its chemokine ligands have been demonstrated in multiple autoimmune disorders, including those that are frequently associated with SS, such as autoimmune thyroiditis, rheumatoid arthritis and SLE [52]. Hence, CXCR3 pathwaymediated enhancement of Th1/Tc1 immune responses in target tissues appears to be a common characteristic and underlying mechanism of SS and its associated autoimmune diseases. A better understanding of the precise contributions of the key players in this process to the disease pathogenesis and persistence could advance the development of new strategies to treat SS and its associated disease conditions.

Acknowledgements We thank Drs. Toshihisa Kawai and Atsushi Ikeda for the assistance in M3R ELISA. This study was supported by the grant from the National Institutes of Health to QY (R01 DE023838)

\section{Compliance with ethical standards}

Conflict of interest The authors declare that they have no conflict of interest.

\section{References}

1. Jin JO, Yu QT. Cell-associated cytokines in the pathogenesis of Sjogren's syndrome. J Clin Cell Immunol. 2013;S!(9):11742

2. Rhodus NL. Sjogren's syndrome. Quintessence Int. 1999;30:689-99.

3. Patel R, Shahane A. The epidemiology of Sjogren's syndrome. Clin Epidemiol. 2014;6:247-55.

4. Fox PC. Autoimmune diseases and Sjogren's syndrome: an autoimmune exocrinopathy. Ann N Y Acad Sci. 2007;1098:15-21.

5. Lee BH, Tudares MA, Nguyen CQ. Sjogren's syndrome: an old tale with a new twist. Arch Immunol Ther Exp. 2009;57:57-66.

6. Voulgarelis M, Tzioufas AG. Pathogenetic mechanisms in the initiation and perpetuation of Sjogren's syndrome. Nat Rev Rheumatol. 2010;6:529-37.

7. Katsifis GE, Moutsopoulos NM, Wahl SM. T lymphocytes in Sjogren's syndrome: contributors to and regulators of pathophysiology. Clin Rev Allergy Immunol. 2007;32:252-64.

8. Singh N, Cohen PL. The T cell in Sjogren's syndrome: force majeure, not spectateur. J Autoimmun. 2012;39:229-33.

9. Hayakawa I, Tedder TF, Zhuang Y. B-lymphocyte depletion ameliorates Sjogren's syndrome in Id3 knockout mice. Immunology. 2007;122:73-9.

10. D'Angelo A, Garzia L, Andre A, et al. Prune cAMP phosphodiesterase binds nm23-H1 and promotes cancer metastasis. Cancer Cell. 2004;5:137-49.

11. Amft N, Bowman SJ. Chemokines and cell trafficking in Sjogren's syndrome. Scand J Immunol. 2001;54:62-9.

12. Groom JR, Luster AD. CXCR3 in T cell function. Exp Cell Res. 2011;317:620-31.

13. Groom JR, Luster AD. CXCR3 ligands: redundant, collaborative and antagonistic functions. Immunol Cell Biol. 2011;89:207-15.

14. Ohmori Y, Wyner L, Narumi S, Armstrong D, Stoler M, Hamilton TA. Tumor necrosis factor-alpha induces cell type and tissuespecific expression of chemoattractant cytokines in vivo. Am J Pathol. 1993;142:861-70.

15. Ohmori Y, Schreiber RD, Hamilton TA. Synergy between interferon-gamma and tumor necrosis factor-alpha in transcriptional activation is mediated by cooperation between signal transducer and activator of transcription 1 and nuclear factor kappaB. J Biol Chem. 1997;272:14899-907.

16. Zhou J, Kawai T, Yu Q. Pathogenic role of endogenous TNFalpha in the development of Sjogren's-like sialadenitis and secretory dysfunction in non-obese diabetic mice. Lab Invest. 2017;97:458-67. 
17. Lacotte S, Brun S, Muller S, Dumortier H. CXCR3, inflammation, and autoimmune diseases. Ann N Y Acad Sci. 2009;1173:310-7.

18. Flier J, Boorsma DM, van Beek PJ, et al. Differential expression of CXCR3 targeting chemokines CXCL10, CXCL9, and CXCL11 in different types of skin inflammation. J Pathol. 2001;194: 398-405.

19. Sorensen TL, Tani M, Jensen J, et al. Expression of specific chemokines and chemokine receptors in the central nervous system of multiple sclerosis patients. J Clin Invest. 1999;103:807-15.

20. Schroepf S, Kappler R, Brand S, et al. Strong overexpression of CXCR3 axis components in childhood inflammatory bowel disease. Inflamm Bowel Dis. 2010;16:1882-90.

21. Katschke KJ Jr., Rottman JB, Ruth JH, et al. Differential expression of chemokine receptors on peripheral blood, synovial fluid, and synovial tissue monocytes/macrophages in rheumatoid arthritis. Arthritis Rheum. 2001;44:1022-32.

22. Frigerio $\mathrm{S}$, Junt $\mathrm{T}$, $\mathrm{Lu} \mathrm{B}$, et al. Beta cells are responsible for CXCR3-mediated T-cell infiltration in insulitis. Nat Med. 2002;8:1414-20.

23. Menke J, Zeller GC, Kikawada E, et al. CXCL9, but not CXCL10, promotes CXCR3-dependent immune-mediated kidney disease. J Am Soc Nephrol. 2008;19:1177-89.

24. Steinmetz OM, Turner JE, Paust HJ, et al. CXCR3 mediates renal Th1 and Th17 immune response in murine lupus nephritis. J Immunol. 2009;183:4693-704.

25. O'Boyle G, Fox CR, Walden HR, et al. Chemokine receptor CXCR3 agonist prevents human T-cell migration in a humanized model of arthritic inflammation. Proc Natl Acad Sci USA. 2012;109:4598-603.

26. Dai Z, Xing L, Cerise J, et al. CXCR3 Blockade Inhibits T Cell Migration into the Skin and Prevents Development of Alopecia Areata. J Immunol. 2016;197:1089-99.

27. Mohan K, Issekutz TB. Blockade of chemokine receptor CXCR3 inhibits $\mathrm{T}$ cell recruitment to inflamed joints and decreases the severity of adjuvant arthritis. J Immunol. 2007;179:8463-9.

28. Herzig DS, Guo Y, Fang G, Toliver-Kinsky TE, Sherwood ER. Therapeutic efficacy of CXCR3 blockade in an experimental model of severe sepsis. Crit Care. 2012;16:R168.

29. Yellin M, Paliienko I, Balanescu A, et al. A phase II, randomized, double-blind, placebo-controlled study evaluating the efficacy and safety of MDX-1100, a fully human anti-CXCL10 monoclonal antibody, in combination with methotrexate in patients with rheumatoid arthritis. Arthritis Rheum. 2012;64:1730-9.

30. Sfriso P, Calabrese F, Grava C, et al. Expression of the interferongamma-inducible $10-\mathrm{kd}$ protein and CXC receptor 3 in the salivary gland lesions of patients with Sjogren's syndrome: comment on the article by Ogawa et al. Arthritis Rheum. 2003;48:2390-1.

31. Ogawa N, Ping L, Zhenjun L, Takada Y, Sugai S. Involvement of the interferon-gamma-induced $\mathrm{T}$ cell-attracting chemokines, interferongamma-inducible 10-kd protein (CXCL10) and monokine induced by interferon-gamma (CXCL9), in the salivary gland lesions of patients with Sjogren's syndrome. Arthritis Rheum. 2002;46:2730-41.

32. Ogawa N, Kawanami T, Shimoyama K, Ping L, Sugai S. Expression of interferon-inducible $\mathrm{T}$ cell alpha chemoattractant (CXCL11) in the salivary glands of patients with Sjogren's syndrome. Clin Immunol. 2004;112:235-8.

33. Hasegawa H, Inoue A, Kohno M, et al. Antagonist of interferoninducible protein 10/CXCL10 ameliorates the progression of autoimmune sialadenitis in MRL/lpr mice. Arthritis Rheum. 2006;54:1174-83.

34. Campanella GS, Tager AM, El Khoury JK, et al. Chemokine receptor CXCR3 and its ligands CXCL9 and CXCL10 are required for the development of murine cerebral malaria. Proc Natl Acad Sci USA. 2008;105:4814-9.

35. Uppaluri R, Sheehan KC, Wang L, et al. Prolongation of cardiac and islet allograft survival by a blocking hamster antimouse CXCR3 monoclonal antibody. Transplantation. 2008;86: 137-47.

36. Youinou P, Devauchelle-Pensec V, Pers JO. Significance of B cells and B cell clonality in Sjogren's syndrome. Arthritis Rheum. 2010;62:2605-10.

37. Kang EH, Lee YJ, Hyon JY, Yun PY, Song YW. Salivary cytokine profiles in primary Sjogren's syndrome differ from those in non-Sjogren sicca in terms of TNF-alpha levels and Th-1/Th-2 ratios. Clin Exp Rheumatol. 2011;29:970-6.

38. Fox RI, Kang HI, Ando D, Abrams J, Pisa E. Cytokine mRNA expression in salivary gland biopsies of Sjogren's syndrome. J Immunol. 1994;152:5532-9.

39. Ewert P, Aguilera S, Alliende C, et al. Disruption of tight junction structure in salivary glands from Sjogren's syndrome patients is linked to proinflammatory cytokine exposure. Arthritis Rheum. 2010;62:1280-9.

40. Baker OJ, Camden JM, Redman RS, et al. Proinflammatory cytokines tumor necrosis factor-alpha and interferon-gamma alter tight junction structure and function in the rat parotid gland Par-C10 cell line. Am J Physiol Cell Physiol. 2008;295: C1191-201.

41. Baker OJ. Tight junctions in salivary epithelium. J Biomed Biotechnol. 2010;2010:278948.

42. Lai Z, Yin H, Cabrera-Perez J, et al. Aquaporin gene therapy corrects Sjogren's syndrome phenotype in mice. Proc Natl Acad Sci USA. 2016;113:5694-9.

43. Culp DJ, Quivey RQ, Bowen WH, Fallon MA, Pearson SK, Faustoferri R. A mouse caries model and evaluation of aqp5-/knockout mice. Caries Res. 2005;39:448-54.

44. Ma T, Song Y, Gillespie A, Carlson EJ, Epstein CJ, Verkman AS. Defective secretion of saliva in transgenic mice lacking aquaporin5 water channels. J Biol Chem. 1999;274:20071-4.

45. Sumida T, Tsuboi $H$, Iizuka M, Hirota $T$, Asashima $H$, Matsumoto I. The role of M3 muscarinic acetylcholine receptor reactive $\mathrm{T}$ cells in Sjogren's syndrome: a critical review. J Autoimmun. 2014;51:44-50.

46. Chaturvedi V, Ertelt JM, Jiang TT, et al. CXCR3 blockade protects against Listeria monocytogenes infection-induced fetal wastage. J Clin Invest. 2015;125:1713-25.

47. Seung E, Cho JL, Sparwasser T, Medoff BD, Luster AD. Inhibiting CXCR3-dependent CD8 $+\mathrm{T}$ cell trafficking enhances tolerance induction in a mouse model of lung rejection. J Immunol. 2011;186:6830-8.

48. Aringer M, Smolen JS. The role of tumor necrosis factor-alpha in systemic lupus erythematosus. Arthritis Res Ther. 2008;10:202.

49. Almoallim H, Al-Ghamdi Y, Almaghrabi H, Alyasi O. Anti-tumor necrosis factor-alpha induced systemic lupus erythematosus. Open Rheumatol J. 2012;6:315-9.

50. Aringer M, Steiner G, Graninger WB, Hofler E, Steiner CW, Smolen JS. Effects of short-term infliximab therapy on autoantibodies in systemic lupus erythematosus. Arthritis Rheum. 2007;56:274-9.

51. Aringer M, Graninger WB, Steiner G, Smolen JS. Safety and efficacy of tumor necrosis factor alpha blockade in systemic lupus erythematosus: an open-label study. Arthritis Rheum. 2004;50:3161-9.

52. Antonelli A, Ferrari SM, Giuggioli D, Ferrannini E, Ferri C, Fallahi P. Chemokine (C-X-C motif) ligand (CXCL)10 in autoimmune diseases. Autoimmun Rev. 2014;13:272-80. 